\title{
El crisol familiar
}

\section{The family melting pop}

\section{Claudia Nicole Castro-Jaramillo ${ }^{a}$, Rosario Barrera-Gálvez ${ }^{b}$, José Arias-Rico ${ }^{c}$, Claudia Teresa Solano-Pérez ${ }^{d}$, Olga Rocio Chávez-Flores ${ }^{e}$, Gwendolyn Samperio-Pelcastre ${ }^{f}$}

\begin{abstract}
:
To understand the family melting pot, it must be recognized that it is family and the influence on the emotional sphere of children. The thought of Daniel Goleman, in his book "Emotional Intelligence", recognizes the family nucleus as the most important for the teaching process - learning of emotional motive for infants, which will have repercussions for the rest of their lives. Emotional development as an existing relationship on the reason for different emotions in children and others.
\end{abstract}

\section{Keywords:}

Family melting pot, emotions, teaching-learning, children's emotional development

\section{Resumen:}

Para comprender el crisol familiar, se debe reconocer que es familia y la influencia en el ámbito emocional de los niños. El pensamiento de Daniel Goleman, en su libro "Inteligencia emocional”, reconoce al núcleo familiar como el más importante para el proceso de enseñanza - aprendizaje de motivo emocional para los infantes, lo cual repercutirá durante el resto de su vida. El desarrollo emocional como relación existente sobre el porqué de distintas emociones en los niños y los demás.

\section{Palabras Clave:}

Crisol familiar, emociones, enseñanza- aprendizaje, desarrollo emocional infantil

\section{Introducción}

El crisol familiar, cómo Goleman lo describe, consiste en el impacto que genera la familia directamente en el desarrollo de la vida emocional del niño, puesto que, el ambiente familiar es la primera escuela donde se desarrolla emocionalmente y determina de forma significativa el grado de inteligencia emocional en el futuro.

\footnotetext{
a Universidad Autónoma del Estado de Hidalgo, Instituto de Ciencias de la Salud, Email: ca414551@uaeh.edu.mx

b Autor de Correspondencia, Universidad Autónoma del Estado de Hidalgo, Instituto de Ciencias de la Salud, https://orcid.org/0000-
} 0002-1949-5424, Email: rosario_barrera@uaeh.edu.mx

${ }^{c}$ Universidad Autónoma del Estado de Hidalgo, Instituto de Ciencias de la Salud, https://orcid.org/0000-0003-0219-0410, Email: josearias.rico@hotmail.com

${ }^{\text {d }}$ Universidad Autónoma del Estado de Hidalgo, Instituto de Ciencias de la Salud, https://orcid.org/0000-0003-4648-981X, Email: claudia_solano@uaeh.edu.mx

e Universidad Autónoma del Estado de Hidalgo, Instituto de Ciencias de la Salud, https://orcid.org/0000-0001-9479-9834, Email: ofloresc@uaeh.edu.mx

${ }^{\mathrm{f}}$ Universidad Autónoma del Estado de Hidalgo, Instituto de Ciencias de la Salud, https://orcid.org/0000-0002-3663-6241, Email: gwendyuni@hotmail.com 
Por ello, cabe desatacar que el proceso de enseñanzaaprendizaje debe ser conciso, para influir positivamente en el niño, enseñándole cómo identificar, interpretar, manejar y utilizar las emociones propias y ajenas. Además, el desarrollo de la empatía, se debe generar en la etapa de la infancia, donde está se adquiere de mejor forma, y en etapas de la vida posteriores, se es muy difícil conseguir.

Brindarle estas bases al infante, repercutirá por el resto de su vida de forma pragmática, por ello la importancia que tiene mantener un crisol familiar adecuado, y así lograr que los niños desarrollen una buena inteligencia emocional, que les ayudará en el ámbito social, académico, psicológico y fisiológico.

\section{El crisol familiar}

Un aspecto de vital importancia respecto al crisol familiar, es entender que es la familia, por tanto, cabe destacar que la Real Academia Española (2001), señala que la familia "es un grupo de personas emparentadas entre sí que viven juntas". Es importante reconocer que este parentesco se obtiene por vínculos de tipo consanguíneo, adopción o matrimonio, donde se incluyen padres, hijos, hermanos, tíos, primos, abuelos, etc.

Se considera a la familia como el espacio que permite en forma integral, a cada individuo, el convivir, crecer y compartir con otras personas, los valores, normas, creencias, tradiciones, comportamientos, conocimientos, experiencias y afectos que resultan indispensables para su pleno desarrollo en la sociedad (Camacho \& Ortiz, 2020, p. 11).

Por consiguiente, la familia y el modo en que esta se maneja, es crucial para el desarrollo de dichas situaciones antes mencionados en los niños, los cuales en el proceso de aprendizaje, se verán en gran porcentaje influenciados por la familia, pues esta será su primer contacto de enseñanza, tanto en aspectos culturales, sociales, psicológicos, mentales y educativos. De las características anteriores, nos centremos en la parte del aprendizaje emocional y las repercusiones en el ámbito educativo conductual, y la importancia de la enseñanza familiar en ese ámbito. Por ende, no se debe limitar la implantación de normas y valores, sino que es necesario formar un ambiente donde se incluyan relaciones familiares saludables para obtener un mejor desarrollo emocional del niño.

Es entonces, donde entra el pensamiento de Daniel Goleman, en su libro "Inteligencia emocional", específicamente en el tema de -El crisol familiar-. Donde la familia, es considerada la primera escuela emocional de los niños, donde estos aprenden a como percibir, comprender, manejar y utilizar los sentimientos y emociones propios y de los demás.
Se reconoce entonces al núcleo familiar como el más importante para el proceso de enseñanzaaprendizaje de motivo emocional para los infantes, lo cual repercutirá durante el resto de su vida. Donde, dicho proceso antes mencionado consiste en una serie de estrategias, métodos, actividades y adquisición de información principalmente, las cuales serán utilizadas para obtener un conocimiento significativo en el niño, que el ayudaran a aprender a cómo afrontar situaciones de la vida diaria, basadas en el conocimiento adquirido.

En este contexto, se puede decir, que tanto el proceso enseñanza- aprendizaje ya antes mencionado, también se ve influenciado por el modelo o los modelos a seguir de los individuos. Donde dichos referentes, además de educar, son un ejemplo que inconscientemente el niño pretende alcanzar o igualar. Por tanto, es indispensable desarrollar el conocimiento con un entorno comunicativo, afectivo y cooperativo, mediante el cual se producirá un aprendizaje permanente y más eficaz y así, se le brindara al infante un ambiente se seguridad y confianza donde pueda desarrollarse de la mejor forma posible en cuestión de salud emocional, la cual incluye factores físicos, psicológicos y sociales.

Esta escuela emocional no solo opera a través de las cosas que los padres dicen o hacen directamente a los niños, sino también en los modelos que ofrecen para enfrentarse a sus propios sentimientos y a los que se produce entre marido y mujer. Algunos padres son dotados maestros emocionales, otros son desastrosos. (Goleman, 2018, p. 224)

Por otro lado, Camacho \& Ortiz (2020) mencionan que:

La calidad del ambiente familiar y las experiencias que mantienen los niños en él son esenciales para su desarrollo emocional. Como en la infancia se aprende más que en cualquier otra etapa de la vida, los niños son sensibles a repetir las acciones que observan a lo largo de su niñez, al momento de crecer los niños imitaran las acciones que observaron y sintieron en su entorno familiar, esencialmente de sus padres, representantes o personas que los cuidaron durante su infancia. (p.22).

Una vez abarcado el ámbito familiar, es necesario reconocer e identificar el término de emoción, pues esta es de las características en que nos basamos. Según Goleman (2018), define a las emociones como:

Impulsos para actuar, planes instantáneos para enfrentarnos a la vida que la evolución nos ha inculcado. La raíz de la palabra emoción es motere, el verbo latino "mover además del prefijo "e" que implica "alejarse", lo que sugiere que en toda emoción hay implícita una tendencia a actuar. (p.17)

La Oxford English Dictionary cuando define el término emoción se refiere a un sentimiento y a los 
pensamientos, los estados biológicos, los estados psicológicos y el tipo de tendencias a la acción que lo caracterizan.

"La emoción refleja el estado emocional bueno o malo en el que se encuentra una persona o la interacción que experimenta" (Chorpita, 2004)

Se entiende que las emociones son aquellas formas particulares de cada individuo para expresarse ante situaciones, contextos, obstáculos específicos y dichas emociones serán expresados basadas en experiencias anteriores, en el cual interviene el proceso enseñanza- aprendizaje, por consiguiente, el manifestar una emoción como consecuencia se tendrá algunas reacciones de carácter fisiológico o conductual.

De forma fisiología puede suceder un aumento de la frecuencia cardiaca, sudoración, nauseas, mareos, respiración agitada, dilatación pupilar, aumento de T/A, etc. Cabe destacar, que en las emociones existen dos tipos; primarias y secundarias, en las emociones primarias encontramos miedo, tristeza, sorpresa, ira o rabia, asco y alegría; en las segundas, son una combinación de las primarias, y como resultado encontramos a la angustia, incertidumbre, preocupación, admiración, asombro, odio, cólera, indignación, enfado, disgusto, soledad, pena, decepción, amor, entusiasmo, etc.

Además, las emociones se ven determinadas por sensaciones, pensamientos, conductas adquiridas que tienen un alto nivel de influencia en el comportamiento e inteligencia desarrollada especialmente en niños, ante esta situación, cabe mencionar que en la etapa de la infancia el desarrollo perceptivo se encuentra activo intensamente, así los niños darán a conocer sus emociones de acuerdo a la forma que han aprendido y los conocimientos adquiridos. Tanto adultos como infantes, suelen experimentar las mismas emociones, y por ello debe tomarse en cuenta la función de estas, y aprender y enseñar a los niños a desarrollar una inteligencia emocional, como ya se había hecho mención, esta consiste en percibir, comprender, manejar y utilizar los sentimientos y emociones propios y de los demás, así mismo, dar el mismo valor de las emociones en niños como de las emociones en un adulto, pues estas son las mismas.

Por ello, se hace hincapié, en que el hogar es la primer escuela para el aprendizaje emocional, y enseñar a los niños que sus emociones son importantes, pero no solo la de ellos, sino también la de los demás, por otra parte, se les debe explicar y preguntar el porqué de dichas emociones que sienten en situaciones concretas, para dar apertura al manejo de las mismas, y de igual forma aprender a no discriminar ningún tipo de emoción.

En base a lo anterior, de acuerdo a Goleman, la relación entre familia- infante, principalmente padres-hijo, dará como resultado lecciones decisivas para la vida del niño, emocionalmente hablando. Además, Goleman a través del análisis de cientos de estudios, identifico como la forma en que los padres tratan y comprender a sus hijos, ya sea con disciplina dura o una comprensión empática y tendrá consecuencias profundas y duraderas en la vida emocional de los hijos.

Al mismo tiempo, Goleman en su libro menciona tres estilos más comunes de paternidad emocionalmente inepta, los cuales son:

1. Ignorar los sentimientos en general, en este tipo de paternidad, los padres tratan las aflicciones emocionales como un problema sin relevancia o aburrido, algo que no es de importancia y se espera a que pase.

2. Mostrarse demasiado liberal, consiste en aquellos padres que se percatan de las emociones del niño, pero afirman que sea cual fuere, la forma en que el niño se enfrente a una tormenta emocional siempre es adecuada, incluso si es, por ejemplo, con golpes.

3. Mostrarse desdeñosos y no sentir respeto por lo que su hijo siente, donde los padres son típicamente desaprobadores, duros tanto en criticas como en sus castigos, dado que existen ciertas prohibiciones de dar a conocer sus emociones.

Sin embargo, Goleman (2018) menciona que "hay padres que aprovechan la oportunidad de un trastorno del hijo para actuar como el equivalente de un mentor o entrenador emocional" (p.225).

De acuerdo a todas estas variaciones entre familia, emociones, enseñanza-aprendizaje, crisol familiar, surge el desarrollo emocional infantil. Dicho desarrollo se refiere a una cuestión sobre la relación existente sobre el porqué de distintas emociones en ellos y los demás. Los niños inician el proceso de reconocimiento de emociones y comienzan a accionar sobre las mismas de acuerdo a las experiencias adquiridas, en esta parte, da inicio la inteligencia emocional del niño y la formación que la familia le ha dado respecto a este tipo de inteligencia, en la cual el niño será capaz o no de identificar, comprender, expresar y regular las emociones, así como pone en pie la empatía de acuerdo a los modelos a seguir que dichos niños tengan.

Henao \& García (2009) mencionan la existencia de "tres aspectos del desarrollo emocional, los cuales permiten conocer de forma más específica la competencia emocional del niño o niña preescolar, a saber: comprensión emocional, capacidad de regulación, y empatía“(p. 789).

La comprensión emocional, entonces se dará de acuerdo a las características individuales da cada niño, las experiencias previas que ha tenido y el contexto familiar 
en el que se desenvuelve, los cuales van a determinar el nivel de complejidad de una situación y como estos serán capaces de comprender y expresar sus emociones, o la forma en que van a actuar de acuerdo a la situación de otro individuo, todo esto, será expresado de acuerdo a lo que este niño cree o a lo que este considere correcto de acuerdo a su ámbito familiar.

La regulación emocional, es desarrollada de acuerdo a las situaciones que viva el niño y la forma en como la familia le enseñara a regular sus emociones. "El niño o niña debe entender el porqué de lo que siente y cuál sería la reacción más adecuada ante esa situación, evaluando la forma más apropiada de enfrentarse a las exigencias externas" (Henao \& Garcia, 2009, p. 791).

El último término del desarrollo emocional, es la empatía, considerada como la capacidad de las personas para comprender una situación emocional de otra y simular el sentimiento o emoción de dicha persona. Durante la infancia, se deben dar lecciones de empatía, por lo contrario, si no se dan, en un futuro será muy difícil que las personas logren generar empatía. Esta empatía comienza desde casa, cuando los padres sintonizan con los sentimientos de los bebés.

Finalmente, cabe destacar la influencia a futuro que tendrá el crisol familiar. Cuando encontramos padres que son emocionalmente inteligentes o expertos, serán padres que toman en cuenta las emociones de sus hijos, cuestionan sobre el porqué están pasando por esas emociones, les ayudan a identificar que sienten, los motivan a regular sus emociones y que estas no les afecten a tal grado de explotar con los demás, son padres que ensañan a sus hijos a reconocer y respetar las emociones ajenas, así como los enseñan a intervenir para manipular dichas emociones de forma positiva, por ejemplo, si un amigo del niño está enojado, los padres lo invitan a que dialogue con su amigo, le ofrezca un estímulo afectivo y lo invite a cambiar de actividad para desechar esa emoción que está produciendo irritación en el niño. Además, los hijos de este tipo de padres, mantienen mejores y más sanas relaciones entre ellos, se muestran más afectuosos y menos tensos respecto a sus padres.

Este tipo de niños, será capaz de identificar y manejar sus emociones. Incluso, al tener mejor desarrollada su inteligencia emocional, presentan niveles de cortisol más reducidos, dicha hormona liberada ante el estrés.

Así mismo, tienen una serie de diversas ventajas sociales, puesto que este tipo de chicos suelen caer mejor a sus pares, e incluso en el ámbito académico, suelen tener una mejor relación docente- alumno, y los maestros suelen considerarlos hábiles socialmente, con un nivel de concentración mayor, y por lo tanto más eficaces.

\section{Conclusión}

El crisol familiar es esencial en el desarrollo emocional del niño. Este crisol familiar corresponde a la primer escuela donde aprenderá bases para la convivencia social, por ende, se afirma que el ambiente padres-hijos debe ser idóneo para un correcto aprendizaje del infante, el cual determinará las pautas de relación con la sociedad en ámbitos de carácter social, educativo, psicológico y cultural.

La inteligencia emocional debe iniciarse y forjarse desde la infancia, enseñarle a los niños a identificar, comprender, manejar y utilizar las emociones propias y ajenas. A largo plazo, se obtienen beneficios para el pequeño. De igual forma, es dispensable fomentar la empatía en esta etapa, pues en etapas siguientes esta será difícil de conseguir.

\section{Referencias}

[1] Camacho, D. C., \& Ortiz, M. E. (2020, 11 febrero). Repositorio Digital ULVR: Influencia de la familia en el desarrollo emocional de los niños de 3 a 4 años de la Escuela Fiscal Guillermo Davis en el periodo lectivo 2018 - 2019. Repositorio Virtual. http://repositorio.ulvr.edu.ec/handle/44000/3575

[2] Goleman, D. (2018). La inteligencia emocional: Por qué es más importante que el cociente intelectual (1.a ed.). Penguin Random House Grupo Editorial.

[3] Henao, G. C., \& García, M. C. (2009). Interacción familiar y desarrollo emocional en niños y niñas. Redalyc. https://www.redalyc.org/pdf/773/77315614009.pdf 